\title{
Frontières
}

\section{Les enjeux liés à la légalisation de l'euthanasie et du suicide assisté au Canada}

\section{Brian L. Mishara}

Volume 20, numéro 1, automne 2007

La « bonne mort»

URI : https://id.erudit.org/iderudit/017947ar

DOI : https://doi.org/10.7202/017947ar

Aller au sommaire du numéro

\section{Éditeur(s)}

Université du Québec à Montréal

ISSN

1180-3479 (imprimé)

1916-0976 (numérique)

Découvrir la revue

Citer cet article

Mishara, B. L. (2007). Les enjeux liés à la légalisation de l'euthanasie et du suicide assisté au Canada. Frontières, 20(1), 47-51.

https://doi.org/10.7202/017947ar
Résumé de l'article

Si on désire légaliser l'euthanasie ou le suicide assisté, on doit préalablement conclure que les méthodes actuelles pour abréger la vie des personnes en phase terminale ne sont pas adéquates. Actuellement, le suicide, le refus de traitement, l'arrêt de traitement et le « double effet » ou le traitement agressif de la douleur même lorsqu'il compromet la durée de vie (dont la sédation terminale est un exemple) sont toutes des activités légales au Canada. Nous soumettons que si jamais d'autres pratiques doivent être légalisées, le suicide assisté devrait être privilégié plutôt que l'euthanasie puisque qu'il s'agit d'une approche plus respectueuse des droits du patient à changer d'avis. L'expérience observée dans les Pays-Bas et en Oregon indique que $40 \%$ des personnes qui demandent l'euthanasie changent d'idée après avoir obtenu les moyens de mettre fin à leurs jours ou après avoir connu un soulagement de la douleur plus adéquat. L'euthanasie et le suicide assisté ne devraient pas se substituer à l'accès à de bons soins palliatifs.
Ce document est protégé par la loi sur le droit d'auteur. L'utilisation des services d’Érudit (y compris la reproduction) est assujettie à sa politique d'utilisation que vous pouvez consulter en ligne.

https://apropos.erudit.org/fr/usagers/politique-dutilisation/ 


\section{Résumé}

Si on désire légaliser l'euthanasie ou le suicide assisté, on doit préalablement conclure que les méthodes actuelles pour abréger la vie des personnes en phase terminale ne sont pas adéquates. Actuellement, le suicide, le refus de traitement, l'arrêt de traitement et le "double effet » ou le traitement agressif de la douleur même lorsqu'il compromet la durée de vie (dont la sédation terminale est un exemple) sont toutes des activités légales au Canada. Nous soumettons que si jamais d'autres pratiques doivent être légalisées, le suicide assisté devrait être privilégié plutôt que l'euthanasie puisque qu'il s'agit d'une approche plus respectueuse des droits du patient à changer d'avis. L'expérience observée dans les Pays-Bas et en Oregon indique que $40 \%$ des personnes qui demandent l'euthanasie changent d'idée après avoir obtenu les moyens de mettre fin à leurs jours ou après avoir connu un soulagement de la douleur plus adéquat. L'euthanasie et le suicide assisté ne devraient pas se substituer à l'accès à de bons soins palliatifs.

Mots clés: euthanasie - suicide assisté suicide - maladie terminale -

soins palliatifs.

\begin{abstract}
In order to legalise euthanasia or assisted suicide one must first conclude that existing methods to foreshorten the life of the terminally ill are not adequate. Currently the practices of suicide, treatment refusal, stopping life-sustaining treatment and the "double effect" or aggressively treating pain symptoms even if life expectancy is compromised (of which terminal sedation is an example) are all legal in Canada. We contend that if other practices are legalized, assisted suicide should be considered rather than euthanasia, since this practice respects better the rights of patients to change their minds. Experiences in the Netherlands and in Oregon indicate that $40 \%$ or more of people who request euthanasia change their minds after obtaining the means or ending their lives or after being offered better treatment of their symptoms. Euthanasia and assisted suicide should not substitute for good palliative care.
\end{abstract}

Keywords: euthanasia - assisted-suicide suicide - terminal illness - palliative care.

\section{Les enjeux liés à la légalisation de l'euthanasie et du suicide assisté au Canada}

\author{
Brian L. Mishara, Ph. D., \\ directeur du Centre de recherche et d'intervention \\ sur le suicide et l'euthanasie (CRISE), professeur \\ au Département de psychologie, UQAM ${ }^{1}$.
}

Cet article présente les enjeux liés à la légalisation de l'euthanasie et du suicide assisté au Canada. Nous décrivons tout d'abord les sept méthodes intentionnelles pour abréger la vie et les aspects légaux impliqués pour chacune de celles-ci. Les méthodes sont comparées afin de pouvoir positionner l'euthanasie et le suicide assisté en relation avec d'autres méthodes légales ou illégales pour abréger la vie d'une personne en phase terminale. Si on veut légaliser l'euthanasie et le suicide assisté, il faut d'abord en arriver à la conclusion que les méthodes légales actuellement disponibles au Canada ne suffisent pas; nous résumons les arguments alors invoqués en faveur de l'euthanasie et du suicide assisté. Nous abordons aussi la question du suicide rationnel et de la rationalité dans la prise de décision de mettre fin à ses jours par euthanasie ou suicide assisté. Nous décrivons les constats observés dans les pays où l'euthanasie et le suicide assisté ont été légalisés et nous citons les résultats de recherches effectuées auprès de personnes atteintes du sida. Nous présentons les différences entre le suicide assisté et l'euthanasie et les implications de ces différences pour la légalisation éventuelle de ces pratiques. Enfin, nous mettons en évidence les liens entre l'euthanasie, le suicide assisté et les soins palliatifs.

\section{LES SEPT MÉTHODES INTENTIONNELLES POUR ABRÉGER LA VIE}

La grande majorité des décès chez l'être humain sont classifiés comme étant imputables à des «causes naturelles» ou "accidentelles». Les morts naturelles et accidentelles ne sont pas considérées comme étant «intentionnelles » parce que la mort est alors non préméditée. On présume dans ces cas que les êtres humains ne jouent pas un rôle direct dans la détermination du moment de leur mort et de 
la façon dont ils vont mourir. Cependant, nous savons aujourd'hui que les comportements des êtres humains peuvent influencer le moment de leur mort "naturelle», ainsi que les façons dont ils vont mourir. Par exemple, les fumeurs présentent un risque beaucoup plus élevé de mourir précocement d'un cancer des poumons que les non-fumeurs. Tout de même, ces risques sont de l'ordre de la probabilité et peuvent être différenciés des méthodes «intentionnelles» d'abrègement de la vie où l'on retrouve un lien causal direct: en effet, dans les décès intentionnels, la mort est imputable directement aux décisions volontaires qui déterminent le moment de la mort ainsi que la façon de mourir.

On peut dénombrer sept méthodes intentionnelles pour abréger la vie:

1) Le suicide: dans le cas d'un suicide, un individu qui a l'intention de mettre fin à ses jours se tue intentionnellement. En général, les personnes suicidaires souffrent d'un trouble mental; au Canada, près de $90 \%$ des personnes qui meurent par suicide peuvent être diagnostiquées comme ayant un trouble mental, généralement un problème psychiatrique de dépression ou des problèmes d'abus d'alcool ou de drogues (Mishara et Tousignant, 2004). Lorsqu'il s'agit d'un suicide, l'intention de mourir a pour but, la plupart du temps, de mettre fin à une souffrance, dans la grande majorité des cas une souffrance psychique que la personne croit inévitable, insupportable et interminable. Le suicide est légal au Canada.

Peu de personnes en phase terminale ou souffrant d'une maladie dégénérative se suicident, par comparaison avec la population en général. Les recherches indiquent que les personnes souffrant d'une maladie terminale ou qui ont un problème dégénératif grave qui se suicident souffrent généralement d'une dépression clinique qui pourrait être traitée ou ne reçoivent pas les traitements appropriés afin d'alléger la douleur physique (Mishara, 1999).

2) Le refus de traitement: les Canadiens ont le droit de refuser toute intervention médicale ou traitement, même si ces traitements sont essentiels à leur survie. Dans le cas d'un refus de traitement, la personne a une mort naturelle. L'intention est différente de l'intention d'une personne suicidaire; en effet, la personne décide ici de ne pas prolonger la vie et de subir le cheminement naturel de la mort sans intervention pour en empêcher ou ralentir le processus.

3) L'arrêt de traitement: depuis le cas célèbre de Nancy B. à Québec (Nancy B. c. Hôtel-Dieu de Québec, 1992), tous les
Canadiens ont le droit de faire cesser les interventions médicales, même celles qui maintiennent une personne en vie. Dans le cas d'un arrêt de traitement, une mort naturelle a lieu habituellement à court terme.

4) Le double effet: il est aussi légal au Canada d'administrer suffisamment de médicaments pour soulager la douleur et les souffrances d'une personne malade, même si ces médicaments auront comme effet secondaire de diminuer l'espérance de vie du patient. Il faut faire ici une distinction importante: l'intention première, quand il y a double effet, est de diminuer la douleur et la souffrance et le fait que l'espérance de vie de l'individu en soit diminuée est un effet secondaire incontournable des traitements qui est accepté par le patient ou son entourage afin de lui permettre de vivre ses derniers jours sans souffrances. Un exemple du double effet qui est relativement peu utilisé au Canada est la sédation terminale. Dans le cas d'une sédation terminale, les interventions ne suffisent pas à soulager les souffrances d'une personne en phase terminale et un médecin décide que la seule façon d'abréger une souffrance intolérable est d'induire chez le patient et avec son accord un état de sommeil profond pour les derniers jours ou les dernières heures de sa vie.

5) L'homicide: lorsqu'un individu tue une autre personne avec ou sans compassion, avec ou sans le désir de la victime de mourir, il s'agit d'un cas d'homicide. L'homicide est illégal au Canada. Un homicide peut être intentionnel ou accidentel. Il est nommément inscrit dans le Code criminel canadien que le fait que la victime voulait mourir ne peut pas être invoqué pour disculper une personne d'avoir commis un homicide.

6) L'euthanasie: l'euthanasie intervient quand un médecin ou une autre personne met fin à la vie d'un individu souffrant d'une maladie terminale ou dégénérative par compassion et à la demande de la personne malade. L'intention du patient est de mourir pour arrêter sa souffrance ou pour vivre une mort qu'elle croit devoir être plus digne. La plupart du temps, dans les pays où l'euthanasie est légale, les personnes souffrant de troubles mentaux ne peuvent y recourir. L'euthanasie est une catégorie de décès qui n'existe pas dans les lois canadiennes. En fait, elle est considérée comme un meurtre au Canada et n'est pas légale. Cette pratique a été légalisée dans les Pays-Bas et en Belgique. Elle a également été légale pendant 9 mois en 1996-1997 dans les territoires du nord de l'Australie.

7) Le suicide assisté: dans le cas d'un suicide assisté, le médecin ou une tierce personne fournit les moyens de se tuer (habituellement, les médicaments létaux) ou les informations sur la manière de se suicider et la personne qui désire mourir se tue elle-même en prenant ces médicaments ou en utilisant les moyens suggérés. L'intention de la personne est de mourir pour faire cesser sa souffrance. Le suicide assisté est illégal au Canada (Gouvernement du Canada, 1985) alors qu'il est légal dans l'État de l'Oregon aux États-Unis et aux Pays-Bas. En Suisse, où il n'a jamais été considéré illégal, il est aussi possible de le pratiquer.

\section{CONDITIONS ET ARGUMENTS POUR UNE LÉGALISATION DU SUICIDE ASSISTÉ OU DE L'EUTHANASIE}

Les conditions et arguments invoqués en faveur d'une légalisation du suicide assisté ou de l'euthanasie sont les suivants.

1) Les autres méthodes déjà existantes pour abréger la vie et qui sont légales au Canada (suicide, refus de traitement, arrêt de traitement et le double effet) ne seraient pas jugées suffisantes pour soulager la souffrance des personnes atteintes d'une maladie en phase terminale.

2) Il serait moralement acceptable, dans certaines situations, d'abréger la vie de personnes souffrant de maladies terminales. Ce qui est moralement acceptable selon les valeurs et croyances religieuses des individus est difficile à débattre parce que les valeurs morales reposent sur la culture, la religion et les expériences de vie des individus. Cependant, l'acceptabilité morale du suicide assisté ou de l'euthanasie est un préalable à la légalisation de ces pratiques.

3) Les bénéfices de la légalisation de ces pratiques justifieraient les risques possibles.

4) Il faudrait déterminer laquelle de ces pratiques de fin de vie doit être admise (suicide assisté, euthanasie ou les deux). Ensuite, il faudrait aussi établir les critères qui permettraient à une personne d'avoir accès au suicide assisté ou à l'euthanasie pour abréger sa vie.

Les personnes qui croient que les méthodes légales pour abréger la vie au Canada ne sont pas suffisantes peuvent baser cette croyance sur plusieurs arguments. Elles peuvent penser que si on ne légalise pas le suicide assisté, il sera plus difficile aux personnes qui désirent se suicider de se procurer les médicaments efficaces à cette fin. Selon cet argument, si on ne légalise pas 
le suicide assisté, les personnes malades qui veulent se tuer risquent de rater leur coup et de vivre avec des séquelles physiques pénibles imputables à la méthode de suicide choisie. Certains constatent également que les méthodes accessibles pour se suicider peuvent s'avérer douloureuses ou faire peur et que nous avons une obligation de rendre plus acceptables les moyens de se suicider de façon «efficace». De même, ceux qui croient que les méthodes actuelles ne suffisent pas disent que le refus et l'arrêt de traitement ne sont pas toujours efficaces pour soulager les souffrances et qu'ils n'entraînent pas la mort assez rapidement. Dans le cas du double effet, certaines personnes assument que les «vraies » raisons pour abréger la vie ne sont pas la diminution de la douleur physique. Pour certains, l'accès à l'euthanasie et au suicide assisté n'est pas simplement une méthode pour mettre un terme à une souffrance physique intolérable et interminable, mais plutôt la facilitation d'une mort «digne» ou un moyen d'aider une personne à déterminer elle-même le moment de sa propre mort.

Les chercheurs en prévention du suicide constatent que le fait de faciliter l'accès à un moyen pour se suicider augmente le risque de suicide. Le fait de rendre sécuritaires les ponts, par exemple par la construction d'une barrière comme celle sur le pont Jacques-Cartier, diminue le nombre de suicides parce que le contrôle d'accès aux moyens qui empêchent les individus de se tuer dans une situation de crise aiguë force les personnes suicidaires à un délai avant de compléter leur suicide (Hawton, 2007). La grande majorité des personnes suicidaires qui doivent ainsi reporter leur passage à l'acte, y compris celles qui souffrent d'une maladie terminale, changent d'avis et ne se tuent pas.

\section{LE SUICIDE: UN CHOIX RATIONNEL ?}

Certaines personnes, par exemple Derek Humphrey $(1986,1991)$ de la Hemlock Society (organisation internationale vouée à la défense du droit à l'euthanasie et au suicide assisté), appuient l'idée selon laquelle un individu est capable de prendre la décision rationnelle de mettre fin à ses jours afin de ne pas prolonger une vie de souffrance et de douleurs. L'idée de décider de s'enlever la vie pour éviter la souffrance est appuyée par plusieurs écoles philosophiques, depuis Épicure et les hédonistes de la Grèce antique, et est élaborée clairement par le philosophe anglais David Hume dans son livre Essays on Suicide (1783). Cependant, la possibilité qu'un suicide puisse être rationnel est un sujet de débat. Certains suicides sont évidemment irrationnels, par exemple, celui du schizophrène qui entend des voix lui commandant de s'enlever la vie. Mais qu'est-ce qui constitue un suicide rationnel?

Le philosophe Jacques Choron (1964) a défini le suicide rationnel comme étant un suicide sans présence de désordre psychiatrique et sans diminution des capacités de raisonnement. Les motivations de cette personne doivent également sembler justifiables ou au moins compréhensibles par la majorité de ses pairs dans la même culture ou dans son groupe social. La première obligation de Choron, soit l'absence de désordre psychiatrique, exclut pour la grande majorité des personnes suicidaires la possibilité de choisir le suicide rationnellement, puisqu'à peu près $90 \%$ des personnes qui meurent par suicide au Canada souffrent d'un désordre psychiatrique lié habituellement à une dépression clinique, à l'alcoolisme ou à l'abus de substances (Lesage, 1994). Même la Hemlock Society qui appuie le suicide rationnel pour les personnes mourantes indique que, si la personne souffre d'un trouble psychique, elle ne doit pas avoir accès au suicide assisté ou à l'euthanasie. Selon Humphrey, il s'agit alors de "suicides émotionnels» ou «homicides de soi irrationnels». La Hemlock Society affirme qu'il ne faut pas encourager le suicide "pour des questions de santé mentale ou des raisons de tristesse». Au Canada, la moitié des personnes qui se suicident consomment de l'alcool (Mishara et Tousignant, 2004) et on peut se demander si on peut exprimer un choix rationnel sous l'influence de drogues ou d'alcool. La grande majorité des personnes en phase terminale prennent des médicaments qui ont des effets importants sur leur état émotionnel et sur leurs habiletés de raisonnement. Est-ce que quelqu'un qui prend des médicaments pour soulager la douleur ou pour arrêter le progrès d'une maladie mais dont les effets secondaires font en sorte que la personne est déprimée ou somnolente peut être considéré apte à prendre des décisions rationnelles?

Mayo (1983), dans une recension des approches philosophiques contemporaines sur le suicide, a défini comme critère d'un suicide rationnel l'habileté de considérer de façon réaliste les choix possibles en relation avec les intérêts fondamentaux de la personne. Mishara (1998) suggère que les décisions les plus importantes prises par les êtres humains sont généralement émotionnelles et très peu rationnelles. Par exemple, on n'utilise pas une pensée rationnelle pour décider qui on va épouser, quelle carrière on va choisir ou même quelle voiture on va acheter. Dans ce contexte, l'auteur se demande comment on peut imaginer que les êtres humains puissent changer leur façon de prendre des décisions lorsqu'ils atteignent la fin de leur vie. Il analyse la situation des personnes en phase terminale et constate que ces individus habituellement souffrent physiquement. En effet, la souffrance physique insupportable est généralement un critère d'accès à l'euthanasie ou au suicide assisté. Il suggère que la souffrance physique intense altère notre capacité à penser rationnellement et à bien analyser la situation puisque la finalité première est la cessation de la souffrance. Il est difficile dans une telle situation de prendre du recul et d'analyser toutes les options.

À cause des problèmes et des confusions soulevés par le concept de «suicide rationnel », David Clarke (1999) a suggéré de remplacer l'idée de suicide rationnel par celle de "suicide compréhensible». Toutefois, les recherches montrent qu'il existe un grand écart entre ce qu'une personne veut faire et ce que les autres croient que cette personne voudrait faire. La plus grande partie de la population peut envisager certaines situations où une personne qui a des handicaps importants serait "mieux morte». La recherche indique cependant très clairement que les personnes qui souffrent d'une maladie terminale ou qui présentent des handicaps sévères ou maladies chroniques sont généralement moins suicidaires que les personnes sans handicap et difficultés (Mishara, 1999).

$\mathrm{Si}$, malgré les objections, on veut accepter l'existence du suicide rationnel dans certaines situations, il faut être capable de spécifier quand un suicide est rationnel et quand il ne l'est pas. On ne peut pas développer des critères fiables liés à la souffrance physique puisque ce qui constitue une souffrance intolérable pour une personne peut s'avérer un niveau d'inconfort acceptable pour une autre personne. De même, il faut déterminer quand un individu est capable de prendre une décision rationnelle et quand il ne l'est pas. Enfin, il faut justifier pourquoi on doit exiger des «décisions rationnelles» dans le cas de l'euthanasie et du suicide assisté quand la grande majorité des décisions importantes des êtres humains sont essentiellement guidées par leurs émotions.

\section{L'EXPÉRIENCE DES PAYS-BAS ET DE L'ÉTAT D'OREGON}

L'expérience observée en Oregon et dans les Pays-Bas nous indique que la décision d'un individu de choisir une mort prématurée par euthanasie et suicide assisté n'est pas une décision stable malgré la force de conviction de l'individu au moment de faire sa demande de mort prématurée. Nous savons que la grande majorité des personnes qui se 
suicident sont très ambivalentes (Mishara et Tousignant, 2004) et que parfois une simple intervention, comme un appel à un centre de prévention du suicide ou bien l'arrivée imprévue d'un ami à la porte, peut faire la différence entre la vie et la mort.

Dans l'État d'Oregon, où le suicide assisté est légal, on compte quand même très peu de demandes de prescriptions de médicaments létaux. En 2003, on dénombrait 30813 morts pour l'année et seulement 67 ordonnances de médicaments létaux prescrites par des médecins et, en 2004, seulement 60 ordonnances létales ont été prescrites pour environ 29600 décès. En 2003, 39 des 67 personnes ont utilisé les médicaments pour se tuer alors qu'en 2004, 37 des 60 personnes ont utilisé ce même moyen. Durant ces deux années, respectivement $43 \%$ et $38 \%$ des personnes qui ont obtenu de telles ordonnances ne s'en sont pas servies pour abréger leur vie. Pour ces personnes donc, il y a eu changement d'avis ou la présence d'autres options.

Dans les Pays-Bas, où se produisent la plupart des décès par euthanasie au monde, entre $50 \%$ et $66 \%$ des demandes sont rejetées par les médecins (Van der Maas et al., 1996) pour la raison que la demande ne correspond pas aux exigences selon lesquelles il faut d'abord tout faire pour abréger la souffrance de la personne avant d'avoir recours à la mort, considérée comme ultime moyen pour mettre un terme à la souffrance. Dans le cas des demandes refusées, on offre au patient des interventions médicales pour diminuer sa douleur ou bien des interventions psychosociales pour diminuer son angoisse psychologique. Il est très rare que ces mêmes personnes formulent une nouvelle demande de mourir par euthanasie lorsque les sources de leurs souffrances sont traitées.

\section{LA STABILITÉ DES DÉCISIONS DE FIN DE VIE DES PERSONNES ATTEINTES DU SIDA}

Nos recherches récentes sur les personnes atteintes du sida indiquent qu'il leur est difficile de faire un choix quant au type de mesures pour abréger ou pour prolonger la vie (Mishara, 2001). Dans une étude de suivi de 101 personnes atteintes du sida à un stade avancé, nous avons trouvé que les intentions mises de l'avant de mettre fin à leurs jours exprimées préalablement n'avaient aucune relation avec les intentions six mois plus tard. Dans les quelques cas où il y a eu des décès chez les participants à cette étude, nous n'avons pu établir de liens entre les décisions prises antérieurement et ce que la personne désirait vraiment à l'approche de sa mort. Dans un cas particulier, la personne prévoyait initialement se suicider avec les médicaments qu'elle avait en mains et elle avait un ami prêt à l'aider s'il le fallait. Cette personne disait qu'elle ne voulait pas être un fardeau pour autrui. Sa maladie avait dégénéré à un point tel que c'était pire que ce qu'elle avait pu imaginer : elle était devenue aveugle, confinée au lit et totalement dépendante au plan physique. Dans ces circonstances et jusqu'aux derniers moments de sa vie, son ami lui a demandé régulièrement si elle désirait qu'on mette en marche son plan d'abréger ses jours par suicide assisté. Cependant, contre toute attente, même dans cette situation grave et incapacitante, cette personne voulait tout faire pour vivre encore quelques jours ou quelques heures et n'avait aucun désir d'enclencher son projet de suicide assisté. Dans un autre cas, une personne avait confirmé qu'elle était croyante et que c'était à Dieu seulement de déterminer le moment de notre mort. Cependant, ayant atteint un état de dépendance et de dégénérescence extrêmes, elle plaidait continuellement pour que son médecin mette fin à ses jours. Nous concluons ainsi que les êtres humains peuvent difficilement prédire ce qu'ils vont ressentir et vouloir lorsqu'ils seront près de mourir.

\section{DIFFÉRENCES ENTRE EUTHANASIE ET SUICIDE ASSISTÉ}

Il existe des différences importantes entre la pratique de l'euthanasie et celle du suicide assisté. Dans la grande majorité des cas de suicide, les personnes suicidaires changent d'avis et ne mettent pas fin à leurs jours (Mishara et Tousignant, 2004). Un très faible pourcentage des personnes sérieusement suicidaires font une tentative; la grande majorité des autres trouvent des solutions à leurs problèmes. Même les personnes qui font des tentatives meurent rarement parce qu'elles arrêtent l'accomplissement de leur plan suicidaire ou font des appels au secours et après coup sont généralement bien contentes d'être encore en vie.

Dans le cas du suicide assisté, la personne décide elle-même si elle veut mourir et quand cette mort surviendra; elle peut changer d'avis à tout moment. Elle n'est pas obligée de prendre les médicaments prescrits par son médecin, qu'elle a en sa possession, et l'expérience en Oregon nous indique qu'environ $40 \%$ des personnes qui ont obtenu les médicaments létaux ne les ont pas utilisés. Cependant, dans les cas d'euthanasie, une personne doit d'abord convaincre un médecin de son droit de mourir. Le cas échéant, à un moment spécifique, un médecin interviendra afin de mettre fin à ses jours. Après de longues démarches et négociations pour con- vaincre les médecins de la légitimité de ce geste, il n'est pas facile de leur demander de reporter l'euthanasie et de revenir le lendemain ou la semaine suivante, ou encore de répéter cette demande plus tard dans le cas où la personne désire reporter son projet d'euthanasie. Dans un tel contexte, il y a une importante pression sociale et une charge émotive tant pour le médecin que pour la personne impliquée. La liberté de changer d'avis peut être compromise à cause de ces pressions sociales. Différemment, dans le cas du suicide assisté, alors que la personne exerce son libre choix de prendre ses médicaments ou non, ces contraintes à la liberté de choisir n'existent pas. Pour ces raisons, si on en vient à légaliser d'autres pratiques de fin de vie que celles actuellement en cours, nous concluons que le suicide assisté est préférable à l'euthanasie.

En général, la population ne fait pas la différence entre l'euthanasie, le suicide assisté et les autres pratiques de fin de vie qui sont tout à fait légales au Canada, soit le refus de traitement, l'arrêt de traitement et le double effet. Une étude récente d'Isabelle Marcoux, chercheure du CRISE (Marcoux et al., 2007), montre qu'un grand nombre des citoyens pensent que la légalisation de l'euthanasie signifie la légalisation de pratiques qui, dans les faits, sont déjà légales, comme le refus et l'arrêt de traitement. Les personnes qui confondent les définitions de l'euthanasie et du suicide assisté sont plus aptes à être en faveur de ces pratiques, selon cette même recherche. Il s'avère donc utile d'instruire la population sur les choix déjà disponibles, sur le droit de refuser et d'arrêter les traitements et le droit d'obtenir des traitements efficaces pour contrôler la souffrance, même si ces traitements diminuaient l'espérance de vie.

On ne peut avoir un débat éclairé qu'avec la participation de citoyens informés. Il faut soigneusement évaluer les avantages du suicide assisté en lieu et place de l'euthanasie. Dans le cas de l'euthanasie, puisque ce seraient surtout les médecins qui seraient appelés à mettre fin à la vie de leur patient, il faudrait s'assurer qu'ils soient prêts à assumer ce rôle. Pendant les 9 mois où l'euthanasie a été légale dans les territoires du Nord de l'Australie en 1996-1997, un seul médecin a accepté de mettre fin aux jours de ses patients. Il a reçu sept demandes et l'a pratiquée sur quatre personnes. De plus, comme il a déjà été mentionné dans le cas du suicide assisté, on peut facilement changer d'avis si on le désire. Les recherches révèlent qu'un grand nombre de personnes, même celles qui vivent des souffrances que la popula- 
tion en général considérerait comme une bonne justification pour mettre fin à ses jours, changent d'avis à la dernière minute et décident de continuer à vivre au lieu de mettre à exécution leur projet de suicide assisté (Mishara et Tousignant, 2004). Dans le cas de l'euthanasie, la liberté de changer d'avis pourrait être compromise par la pression sociale créée lorsqu'un médecin, convaincu que la personne a choisi rationnellement de mourir, se présente afin d'administrer l'euthanasie.

Pour résumer notre discussion, avant d'appuyer la légalisation de l'euthanasie ou du suicide assisté, il convient d'analyser soigneusement si les méthodes légales pour abréger la vie actuellement disponibles au Canada suffisent pour répondre aux besoins des personnes souffrantes et atteintes d'une maladie terminale. Très souvent, les citoyens qui sont en faveur de la légalisation de l'euthanasie ou du suicide assisté confondent ces pratiques avec celles qui ont cours actuellement et qui sont déjà légales, à savoir le refus de traitement, l'arrêt de traitement ou le double effet pour le soulagement de la douleur. Si on se fie à l'expérience de l'Oregon, on peut prédire que seulement une très petite proportion des personnes mourantes en viendra à demander un suicide assisté et à peu près $40 \%$ de celles qui auront obtenu des médicaments changeront d'avis. L'expérience de l'Oregon démontre également que l'ambivalence face au suicide, qui fait en sorte que la grande majorité des personnes suicidaires changent d'avis et abandonnent leur projet de se suicider, est également présente chez les personnes en phase terminale désireuses d'avoir accès au suicide assisté. Pour cette raison, ainsi qu'à cause des dangers de compromettre la liberté de changer d'avis dans la situation où l'euthanasie est pratiquée par un médecin, si on en arrive à légaliser une autre pratique pour abréger la vie au Canada, on doit favoriser le suicide assisté plutôt que l'euthanasie.

Si le Canada adopte une loi sur le suicide assisté ou l'euthanasie, il devrait également protéger les citoyens contre les pressions pour choisir la mort parce que d'autres façons de diminuer la souffrance ne sont pas disponibles ou sont moins facilement accessibles. Il faut reconnaître qu'il est très rare que les citoyens revendiquent une mort prématurée, même dans les pays où le suicide assisté et l'euthanasie sont légalisés. Avant tout, on devrait assurer l'accès à des soins physiques et psychosociaux adéquats, y compris des soins palliatifs de qualité à l'ensemble des citoyens. Est-ce que nous voulons vivre dans une société où les personnes qui souffrent ont le choix entre continuer à souffrir ou mettre fin à leurs souffrances en mourant par suicide assisté ou euthanasie, cela parce qu'elles sont sur une liste d'attente pour les soins palliatifs? En général, selon les recherches, les personnes suicidaires et souffrant de maladies terminales trouvent d'autres solutions pour abréger leurs souffrances, sans avoir recours à une mort prématurée. Il est essentiel que la mort par suicide assisté ou par euthanasie ne soit pas simplifiée au point qu'elle soit plus facile à obtenir que de bons traitements pour soulager la souffrance.

\section{Bibliographie}

CHORON, J. (1964). Modern Man and Mortality, New York, The Macmillan Company.

CLARKE, D.M. (1999). «Autonomy, rationality and the wish to die ", Journal of Medical Ethics, vol. 25, n 6, p. 457-462.

GOUVERNEMENT DU CANADA (1985). Code criminel, L. R.(1985), ch. C-46, art. 241, Ottawa.

HAWTON, K. (2007). « Restricting access to methods of suicide: Rationale and evaluation of this approach to suicide prevention », CRISIS (The Journal of Crisis Intervention and Suicide Prevention), vol. 28, suppl. 1, p. 4-9.

HUME, D. (1783). Essays on Suicide and the Immortality of the Soul, Whitefish (MT), Kessinger Publishing, LLC.

HUMPHREY, D. (1999). Final Exit, Eugene (OR), The Hemlock Society.

HUMPHREY, D. (1986). "The case for rational suicide», Euthanasia Review, vol. 1, $\mathrm{n}^{\circ} 3$, p. 172-176.

LESAGE, A. (1994). "Troubles mentaux et suicide», Santé mentale au Québec, vol. 19, $\mathrm{n}^{\circ} 2$, p. 7-14.

MARCOUX, I., B.L. MISHARA et C. DURAND (2007). "Confusion between euthanasia and other end of life decisions: Influences on public opinion poll results ", Canadian Journal of Public Health, vol. 98, $n^{\circ} 3$, p. 235-239.

MAYO, D.J. (1983). «Contemporary philosophical literature on suicide: A review», Suicide and Life-Threatening Behavior, vol. 13, no 4, p. 313-345.

MISHARA, B.L. (2001). Why Persons with AIDS Choose to Live or Die, présentation dans le cadre du XII ${ }^{\mathrm{e}}$ Congrès de l'Association canadienne pour la prévention du suicide, St. John's (Terre-Neuve), le 26 octobre 2001.

MISHARA, B.L. (1999). "Synthesis of research and evidence on factors affecting the desire of terminally ill or seriously chronically ill persons to hasten death", Omega, International Journal on Death and Dying, vol. $39, \mathrm{n}^{\circ} 1$, p. 1-70.

MISHARA, B.L. (1998). «The right to die and the right to live: Perspectives on euthanasia and assisted suicide", dans A. LEENAARS, M. KRAL, R. DYCK et S. WENCKSTERN (dir.), Suicide in Canada, Toronto, University of Toronto Press, p. 441-458.
MISHARA, B.L. ET M. TOUSIGNANT (2004). Comprendre le suicide, Montréal, Presses de l'Université de Montréal.

NANCY B. c. HÔTEL-DIEU DE QUÉBEC (1992). R.J.Q. 361 (C.S).

OREGON DEPARTMENT OF HUMAN SERVICES (2005). Seventh Annual Report on Oregon's Death With Dignity Act, État de l'Oregon.

OREGON DEPARTMENT OF HUMAN SERVICES (2004). Sixth Annual Report on Oregon's Death With Dignity Act, État de l'Oregon.

VAN DER MAAS, P. J., G. VAN DER WAL, I. HAVERKATE, C.L.M. DE GRAAF, J.G.C. KESTER, B.D. ONWUTEAKAPHILIPSEN et al. (1996). "Euthanasia, physician-assisted suicide, and other medical practices involving the end of life in the Netherlands, 1990-1995 », New England Journal of Medicine, vol. 335, $\mathrm{n}^{\circ} 1$, p. 699-705.

\section{Note}

1. L'auteur tient à remercier Jean-Yves Boucher, François Chagnon, Éveline Pilon et Steve Carrière pour leur lecture critique d'une version préliminaire de cet article. 